\title{
Primary Effusion Lymphoma: Is Dose-Adjusted-EPOCH Worthwhile Therapy?
}

\author{
Kegan Jessamy ${ }^{a} \quad$ Fidelis O. Ojevwe $^{a} \quad$ Ravi Doobay $^{a} \quad$ Rana Naous $^{b}$ \\ John $\mathrm{Yu}^{\mathrm{c}}$ Sheila M. Lemke \\ a Department of Medicine, SUNY Upstate Medical University, Syracuse, N.Y., USA; \\ ${ }^{b}$ Department of Pathology, SUNY Upstate Medical University, Syracuse, N.Y., USA; \\ ${ }^{c}$ College of Medicine, SUNY Upstate Medical University, Syracuse, N.Y., USA; ${ }^{d}$ Department \\ of Medicine, Hematology/Oncology Division, SUNY Upstate Medical University, \\ Syracuse, N.Y., USA
}

\section{Keywords}

Primary effusion lymphoma - Human immunodeficiency virus - Acquired immunodeficiency syndrome $\cdot$ Kaposi's sarcoma $\cdot \mathrm{EPOCH} \cdot$ Dose-adjusted EPOCH

\begin{abstract}
Primary effusion lymphoma (PEL) is a rare condition, which accounts for approximately $4 \%$ of all human immunodeficiency virus (HIV)-associated non-Hodgkin lymphomas. PEL has a predilection for body cavities and occurs in the pleural space, pericardium, and peritoneum. Without treatment, the median survival is approximately 2-3 months, and with chemotherapy, the median survival is approximately 6 months. We describe the case of a 47-year-old male with HIV and Kaposi's sarcoma who presented with complaints of abdominal pain and distension and was subsequently diagnosed with PEL. Despite limited clinical data being available, chemotherapy with dose-adjusted etoposide, prednisone, vincristine, cyclophosphamide, and doxorubicin (EPOCH) has proven to increase survival rates in patients with this condition.




\section{Case Reports in Oncology}

\section{Introduction}

Primary effusion lymphoma (PEL) is a rare disease, which accounts for approximately $4 \%$ of all human immunodeficiency virus (HIV)-associated non-Hodgkin lymphomas (NHL) [1]. PEL has a unique clinical presentation with a predilection for body cavities such as the pleural space, pericardium, and the peritoneum. Extracavitary tumors are rare in PEL and may be histologically indistinguishable from PEL [2]. By definition, PEL cases must show evidence of infection with Kaposi's sarcoma (KS)-associated herpesvirus-8 (HHV-8) [1].

The development of NHL in a patient infected with HIV was declared an acquired immunodeficiency syndrome (AIDS)-defining illness in 1985 and since then, it has been approximated that between 5 and $20 \%$ of all HIV-positive patients will develop NHL at some point during their lifetime [1]. The mechanism of the proliferation of the disease with HHV-8 remains uncertain [3]. Since the introduction of highly active antiretroviral therapy (HAART) in the 1990's, the incidence of AIDS-related NHL has decreased. However, given its rarity, the prognosis of PEL remains poor [4]. Much like other NHLs, combination chemotherapy [such as cyclophosphamide, doxorubicin, vincristine, and prednisone (CHOP)], combined with HAART has been the cornerstone for the treatment of PEL. Nevertheless, durable responses are rarely seen, and survival is short with median survivals of around 4-6 months [2]. An alternative therapy consisting of etoposide, prednisone, vincristine, cyclophosphamide, and doxorubicin (EPOCH) with dose adjustment (DA-EPOCH) has been reported to provide a favorable prognosis [5].

\section{Case Presentation}

A 47-year-old male presented to our institution complaining of a 1-month history of abdominal pain. His medical history was significant for HIV (diagnosed 18 years ago), KS, and chronic left-leg edema secondary to pelvic infiltration of KS, compressing the common femoral and superficial femoral veins for which he previously received chemotherapy (paclitaxel, doxorubicin, and then gemcitabine) and electron beam radiation. The patient also noted worsening abdominal distention, which he attributed to his worsening abdominal pain. In addition to his abdominal distension, he gave a history of unintentional $4.5 \mathrm{~kg}$ (10 lbs) weight loss over a 1-week period and bilateral lower-extremity edema that was worse on the left side. He also complained of progressively worsening dyspnea, nausea, intermittent pyrexia as high as $38.3^{\circ} \mathrm{C}\left(101^{\circ} \mathrm{F}\right)$, anorexia, rhinorrhea, and malaise for 2 days prior to admission. He admitted to being compliant with his current antiretroviral regimen, which included raltegravir, ritonavir, and the combination abacavir/lamivudine/zidovudine.

On admission, his blood pressure was $113 / 77 \mathrm{~mm} \mathrm{Hg}$, pulse 97 beats per minute, temperature $36.5^{\circ} \mathrm{C}\left(97.7^{\circ} \mathrm{F}\right)$, respiratory rate 20 breaths per minute, and pulse oximetry $99 \%$ (room air). Physical examination was significant for generalized abdominal distension on inspection, decreased frequency of bowel sounds, and generalized tenderness to palpation without any rebound tenderness or guarding. He had bilateral flank dullness to percussion, positive shifting dullness, and fluid thrill. There was no cervical, axillary, epitrochlear, and inguinal adenopathy. HIV serology showed an absolute CD4 count of $261 \mathrm{cells} / \mu \mathrm{l}$ and a quantitative HIV-1 RNA level of 220 copies/ml. A CT scan of the abdomen and pelvis was significant for moderate-volume abdominal ascites prominently seen in the perihepatic region extending into the right paracolic gutter without any evidence of bowel obstruction (fig. 1). His ascites rapidly reaccumulated within $48 \mathrm{~h}$ after the initial paracentesis. 


\section{Case Reports in Oncology}

His initial ultrasound-guided paracentesis yielded 1,365 ml cloudy, yellow ascitic fluid. Analysis of the ascitic fluid was positive for a large number of atypical lymphoid cells. Flow cytometry cytospin showed pleomorphic, large lymphoid cells with large nuclei, multiple prominent nucleoli, and deeply basophilic cytoplasm with a plasmacytoid appearance. Polymerase chain reaction showed clonal B cells, and HHV-8 immunostain revealed positive nuclear staining (fig. 2), which confirmed the diagnosis of PEL. The cell block preparation revealed multiple malignant lymphocytes (fig. 3).

Given his Eastern Cooperative Oncology Group performance score of 1, he was promptly started on DA-EPOCH as we were optimistic of a good outcome. His ascites gradually improved and did not recur on follow-up visits after 4 cycles of DA-EPOCH.

\section{Discussion}

PEL is clinically characterized by lymphomatous effusions in body cavities, usually without extracavitary tumor masses, with clinical symptoms depending on the cavities involved. The most common sites are the peritoneal, pleural, and pericardial cavities. Joint and meningeal spaces are rarely involved [1]. The clinical presentation may involve abdominal distension from ascites, or dyspnea from pleural or pericardial effusion due to the mass effect of malignant effusion. The most common causes of death in patients with PEL are lymphoma progression, opportunistic infection, and HIV-related complications [6].

The definitive diagnosis of PEL is made from the detection of viral infection with HHV-8 in the neoplastic cells [7]. Cytologic examination of fluid demonstrates large cells with either an immunoblastic or plasmablastic appearance [8]. Immunohistochemistry to detect latency-associated nuclear antigen, LANA-1 (fig. 2), is currently the standard method to demonstrate evidence of KS herpesvirus/HHV-8 in lymphoma cells [6]. Viral interleukin 6 is expressed by a variable subset of lymphoma cells, and immunohistochemical studies for this protein may be helpful for confirmation [6].

Without treatment, the median survival after diagnosis is approximately 2-3 months [9, 10]. A multicenter, retrospective series of 28 patients on chemotherapy showed a median survival of 6.2 months and a 1-year overall survival rate of 39.3\% [4]. PEL is rare, and there are few longitudinal observational series of patients and prospective randomized clinical trials. Therefore, treatment is usually guided based on case reports and expert consensus opinions [5]. Patients should always be encouraged to enroll in clinical trials whenever or wherever available [1]. Traditional chemotherapy with CHOP is a common regimen for treating NHL and has been attempted for the treatment of PEL. Despite the availability of chemotherapeutic regimens, the prognosis remains poor [5].

Infusion therapy with EPOCH has been shown to be a well-tolerated and effective treatment [5]. DA-EPOCH chemotherapy evolved from the hypothesis that incremental improvements in CHOP chemotherapy via scheduling, drug selection, and pharmacokinetic optimization can improve the outcome in patients with aggressive lymphomas [11]. DA-EPOCH was shown to produce an improved progression-free survival and overall survival when compared with CHOP or CHOP-based regimens [11].

DA-EPOCH infusion therapy should be administered through central venous access. Inpatients often administered DA-EPOCH may receive the 96-hour infusions on an outpatient basis, utilizing a portable infusion pump [11] (table 1). 


\section{Case Reports in Oncology}

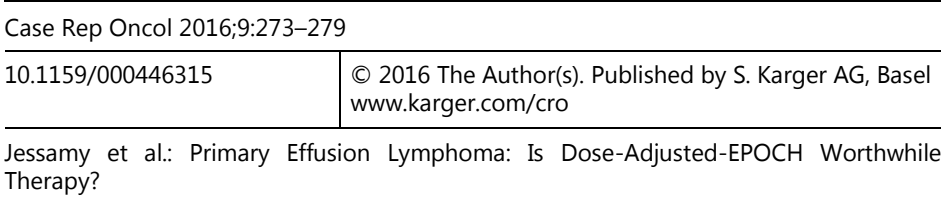
Therapy?

\section{DA-EPOCH [11]}

Complete blood counts are obtained twice weekly. Dose escalation above the starting doses in the case of good chemotherapy tolerability applies simultaneously to etoposide, doxorubicin, and cyclophosphamide. Dose de-escalation below the starting doses in the case of poor patient tolerance applies to cyclophosphamide only. If the absolute neutrophil count (ANC) nadir is $>500 / \mu \mathrm{l}$, the doses of etoposide, doxorubicin, and cyclophosphamide for the next cycle are all increased by $20 \%$. If the ANC nadir is $<500 / \mu$ on 1 or 2 blood checks but the ANC rises $>500 / \mu \mathrm{l}$ at the time of the third check (i.e., the duration of agranulocytosis is $<9$ days) and the nadir of the platelet count is $>25,000 / \mu \mathrm{l}$, the dose for the next course will remain the same. If the ANC nadir is $<500 / \mu \mathrm{l}$ for $\geq 10$ days, or if the nadir of the platelet count at every time falls below $25,000 / \mu \mathrm{l}$, the doses of etoposide, doxorubicin, and cyclophosphamide are reduced by $20 \%$ below the doses used in the previous cycle, but doxorubicin and etoposide should not be reduced below the dosage given in the first course (table 2).

Single case reports have shown complete remissions of PEL after implementation of HAART, without chemotherapeutic drugs $[12,13]$. Initiating or continuing HAART as part of supportive therapy is recommended when commencing treatment for HIV-positive patients with PEL. The routine use of growth factors, such as granulocyte colony-stimulating factor, to avoid prolonged periods of neutropenia from chemotherapy is standard practice for all AIDS-related lymphoma patients [14]. An anti-CD20 monoclonal antibody, rituximab (R), has been added to the EPOCH regimen (R-EPOCH) in rare PEL cases with CD20 expression [14]. An analysis comparing the treatment regimens for AIDS Malignancy Consortium (AMC) Trials AMC010 and AMC034 showed that patients treated with R-EPOCH had improved eventfree and overall survival rates [15]. Unfortunately, regardless of treatment, there is no evidence of achieving cure of PEL with conventional systemic chemotherapy [2].

\section{Conclusion}

PEL is a rare and highly aggressive malignancy with poor outcomes. Unfortunately, there are few longitudinal observational studies or randomized clinical trials, and treatment is largely guided by expert consensus. Despite limited clinical data being available, chemotherapy and HAART have proven to increase survival rates amongst patients with this condition. As evidenced by the lack of recurrence of ascites in our patient, DA-EPOCH is an effective chemotherapeutic regimen with proven improved overall survival.

\section{Statement of Ethics}

The authors have no ethical conflicts to disclose.

\section{Disclosure Statement}

The authors have no conflicts of interest to declare.

\section{References}

1 Chen Y, Rahemtullah A, Hochberg E: Primary effusion lymphoma. Oncologist 2007;12:569-576 8p. 


\section{Case Reports in Oncology}

\begin{tabular}{l|l}
\hline Case Rep Oncol 2016;9:273-279 \\
\hline $10.1159 / 000446315$ & $\begin{array}{l}\text { (c) 2016 The Author(s). Published by S. Karger AG, Basel } \\
\text { www.karger.com/cro }\end{array}$ \\
\hline
\end{tabular}

Jessamy et al:: Primary Effusion Lymphoma: Is Dose-Adjusted-EPOCH Worthwhile Therapy?

-2 El-Ayass W, Yu E, Karcher D, Aragon-Ching J: Complete response to EPOCH in a patient with HIV and extracavitary primary effusion lymphoma involving the colon: a case report and review of literature. Clin Lymphoma, Myeloma Leuk 2012;12:144-147.

-3 Millet A, Singh S, Gittens-Backus G, Dang K, Shokrani B: An unusual case of invasive Kaposi's sarcoma with primary effusion lymphoma in an HIV positive patient: case report and literature review. Case Rep Oncol Med 2015;2015:789616.

4 Boulanger E, Gerard L, Gabarre J, et al: Prognostic factors and outcome of human herpesvirus 8associated primary effusion lymphoma in patients with AIDS. J Clin Oncol 2005;23:4372-4380.

-5 Okada S, Goto H, Yotsumoto M: Current status of treatment for primary effusion lymphoma. Intractable Rare Dis Res 2014;3:65-74.

6 Patel S, Xiao P: Primary effusion lymphoma. Arch Pathol Lab Med 2013;137:1152-1154.

7 Said J, Cesarman E: Primary effusion lymphoma; in Swerdlow SH, Campo E, Harris NL, Jaffe ES (eds): WHO Classification of Tumours of Haematopoietic and Lymphoid Tissues, ed 4. Lyon, International Agency for Research on Cancer, 2008, pp 260-261.

8 Schulz TF: The pleiotropic effects of Kaposi's sarcoma herpesvirus. J Pathol 2006;208:187-198.

-9 Valencia Ortega M, Martínez Santos P, González Lahoz J, et al: Primary cavity-based lymphoma and HIV infection (in Spanish). Rev Clin Esp 1999;199:73-77.

10 Komanduri K, Luce J, McGrath M, Herndier B, Ng V: The natural history and molecular heterogeneity of HIV-associated primary malignant lymphomatous effusions. J Acquir Immune Defic Syndr Hum Retrovirol 1996;13:215-226.

11 Wilson W, Grossbard M, Balis F, et al: Dose-adjusted EPOCH chemotherapy for untreated large B-cell lymphomas: a pharmacodynamic approach with high efficacy. Blood 2002;99:2685-2693.

12 Hocqueloux L, Agbalika F, Oksenhendler E, et al: Long-term remission of an AIDS-related primary effusion lymphoma with antiviral therapy. AIDS 2001;15:280-282.

-13 Oksenhendler E, Clauvel JP, Jouveshomme S, et al: Complete remission of a primary effusion lymphoma with antiretroviral therapy. Am J Hematol 1998;57:266.

14 Carbone A, Gloghini A: KSHV/HHV8-associated lymphomas. Br J Haematol 2008;140:13-24

15 Barta S, Lee J, Kaplan L, Noy A, Sparano J: Pooled analysis of AIDS malignancy consortium trials evaluating rituximab plus CHOP or infusional EPOCH chemotherapy in HIV-associated non-Hodgkin lymphoma. Cancer 2012;118:3977-3983.

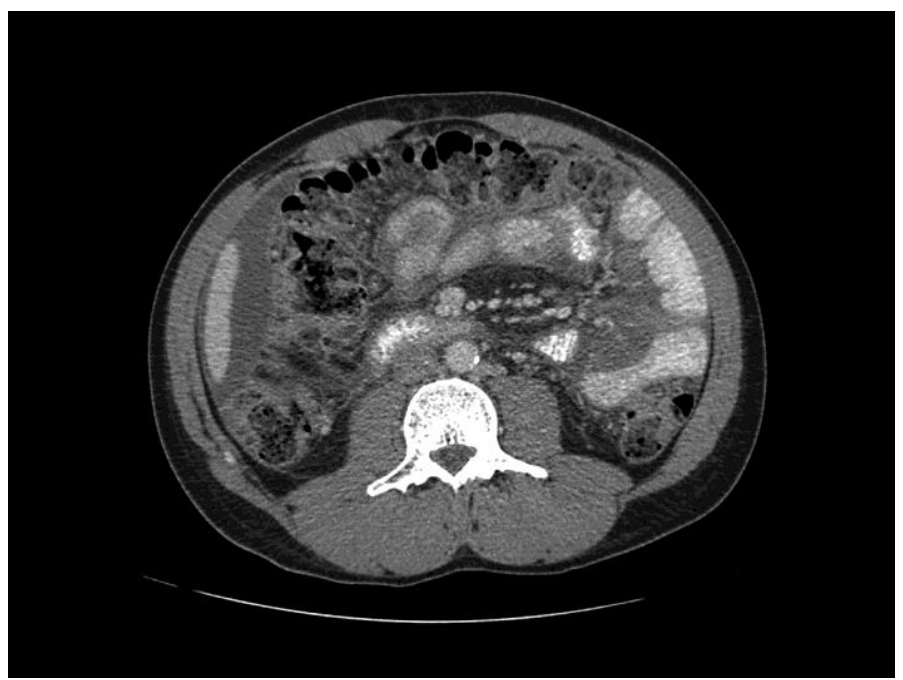

Fig. 1. CT scan of the abdomen and pelvis with abdominal ascites. 


\section{Case Reports in Oncology}

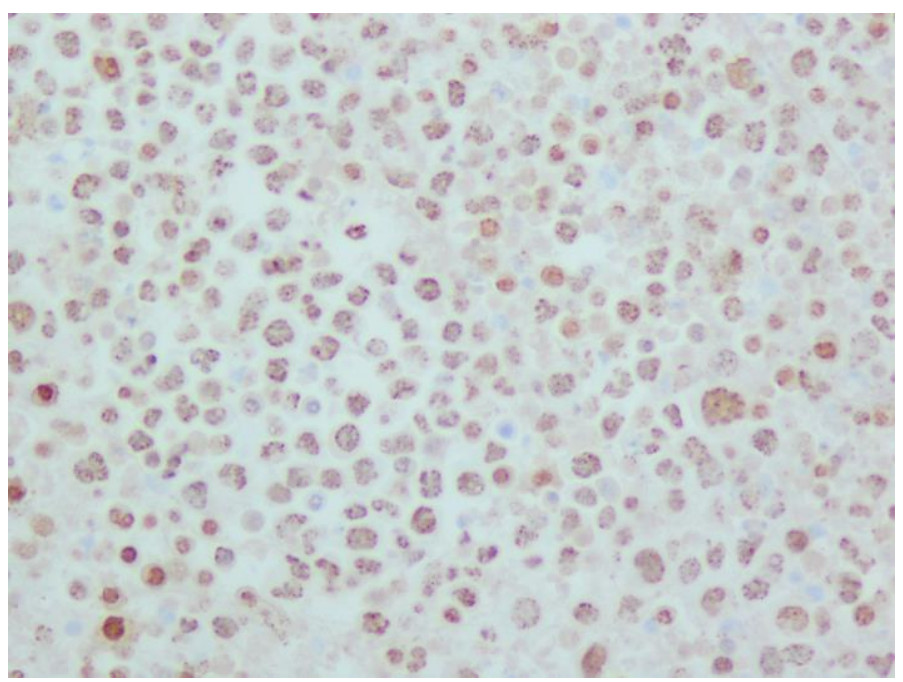

Fig. 2. HHV-8 immunostain showing positive nuclear staining. HHV-8 is required for an unequivocal diagnosis of PEL.

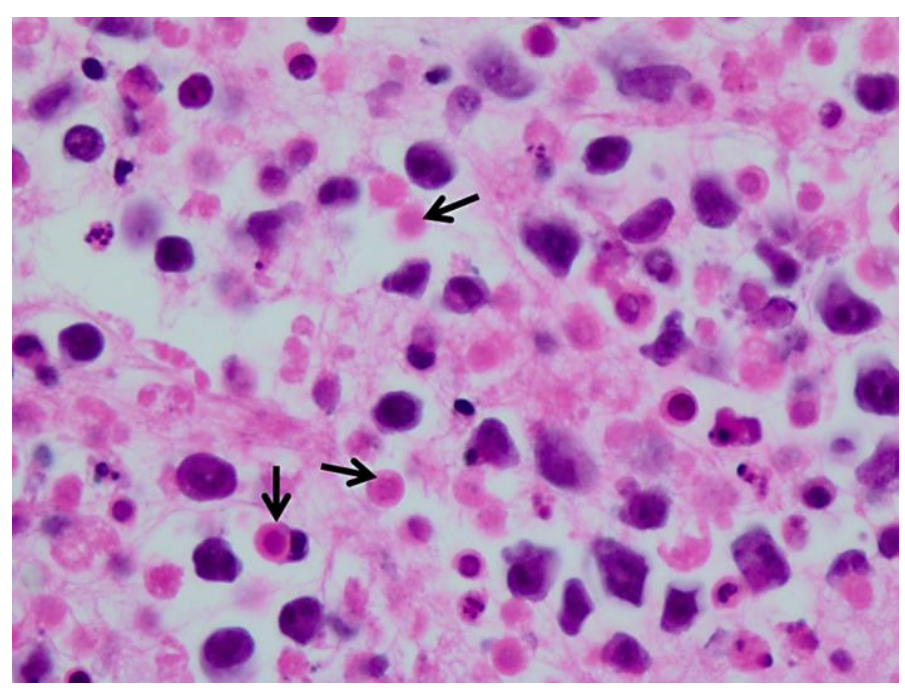

Fig. 3. High-power view of the cell block highlighting background single-cell necrosis (arrows) admixed with the malignant lymphocytes. H\&E. $\times 40$. 
Table 1. EPOCH starting dose level (level 1)

\begin{tabular}{llll}
\hline Drug & Dose & Route & Treatment days \\
\hline $\begin{array}{lll}\text { Infused agents } \\
\quad\end{array}$ & & \\
$\quad$ Etoposide & $50 \mathrm{mg} / \mathrm{m}^{2} /$ day & CIV & $1,2,3,4$ \\
$\quad$ Doxorubicin & $10 \mathrm{mg} / \mathrm{m}^{2} /$ day & CIV & $1,2,3,4$ \\
$\quad$ Vincristine & $0.4 \mathrm{mg} / \mathrm{m}^{2} /$ day & CIV & $1,2,3,4$ \\
Bolus agents & & & \\
$\quad$ Cyclophosphamide & $750 \mathrm{mg} / \mathrm{m}^{2} /$ day & IV & 5 \\
$\quad$ Prednisone & $60 \mathrm{mg} / \mathrm{m}^{2} /$ day & Oral & $1,2,3,4,5$ \\
$\quad$ G-CSF & $5 \mu \mathrm{kg} /$ day & SC & 6 to ANC $>5 \times 10^{9} / \mathrm{l}$ past nadir \\
Next cycleb & & & Day 21 \\
\hline
\end{tabular}

G-CSF = Granulocyte colony-stimulating factor; $\mathrm{CIV}=$ continuous intravenous; IV = intravenous, $\mathrm{SC}=$ subcutaneous. a Etoposide, doxorubixin, and vincristine could be mixed in the same solution. b Begin on day 21, if the ANC is at least $1 \times 10^{9} / \mathrm{l}$ and the platelet count is at least $100 \times 10^{9} /$ l. Adapted from Wilson et al. [11].

Table 2. DA-EPOCH paradigm

\begin{tabular}{|c|c|}
\hline Nadir measurements ${ }^{a}$ & Dose adjustment ${ }^{b}$ \\
\hline If ANC nadir at least $0.5 \times 10^{9} / \mathrm{l}$ & $\begin{array}{l}\text { Dose of etoposide, doxorubicin, and } \\
\text { cyclophosphamide } 20 \% \text { above that of last cycle }\end{array}$ \\
\hline If ANC nadir $<0.5 \times 10^{9} / \mathrm{l}$ on 1 or 2 measurements & Same dose(s) as last cycle \\
\hline If ANC nadir $<0.5 \times 10^{9} / \mathrm{l}$ on at least 3 measurements & $\begin{array}{l}\text { Dose of etoposide, doxorubicin, and } \\
\text { cyclophosphamide } 20 \% \text { below that of last cycle }\end{array}$ \\
\hline If nadir of platelet count $<25 \times 10^{9} / \mathrm{l}$ on 1 measurement & $\begin{array}{l}\text { Dose of etoposide, doxorubicin, and } \\
\text { cyclophosphamide } 20 \% \text { below that of last cycle }\end{array}$ \\
\hline
\end{tabular}

